\title{
Thermodynamics of Irreversible Processes across a Boundary: Elementary Principles
}

\author{
Jurgen M. Honig \\ Department of Chemistry, Purdue University \\ 560 Oval Drive, West Lafayette, IN, 47907-2084, USA
}

Tel: 1-765-494-5279 E-mail:jmh@purdue.edu

Received: August 17, 2011

Accepted: September 6, 2011 Published: November 1, 2011

doi:10.5539/apr.v3n2p3

URL: http://dx.doi.org/10.5539/apr.v3n2p3

\begin{abstract}
The commonly used thermodynamic variables are generalized so as to take account of irreversible processes across a thin boundary separating a system from its surroundings. This permits one to relate heat transfer and work performance in irreversible processes to those carried out reversibly. The commonly used functions of state have been generalized to include the contribution of irreversible changes to the entropy of the system. These contributions are specified in terms of experimental observables.
\end{abstract}

Keywords: Quasistatic irreversible processes, System-surrounding interactions across a thin boundary, Generalized thermodynamic functions of state, Specification of entropy associated with irreversibility

\section{Basic Commentary}

The magnificent structure of thermodynamics has been of immense utility in unifying our understanding of physical phenomena for over 170 years. The early developments that were based on the fundamental laws of thermodynamics involved the concept of equilibrium and reversibility: systems were assumed to be either in a stationary state, or all processes were assumed to proceed infinitely slowly so that the system never deviated significantly from equilibrium conditions. Beginning around the turn of the $20^{\text {th }}$ century, investigators extended the original theories so as to include processes that involved first order departures from the equilibrium state, by allowing the properties of interest to become local functions of position and time. This opened up a plethora of new effects and applications under the heading of nonequilibium thermodynamics or extended thermodynamics, which are well documented in many reviews, a selection of which is provided below (Meixner, 1954) (de Groot and Mazur, 1962) (Prigogine, 1967) (Gyarmati, 1970) (Haase, 1990) (Mueller and Ruggeri, 1996) (Jou, 1996) (Kondepudi and Prigogine, 1998) (Honig, 2007). In this connection it may be of interest to draw attention to a recent complementary approach (Honig and Ben Amotz, 2005, 2008) (Ben Amotz and Honig, 2006), in which the thermodynamic functions of state are enlarged to accommodate the contribution of irreversible processes to the entropy of the system. This is achieved by replacing the customary inequalities in elementary treatments by equalities. Such an approach finds its principal application in dealing with the transfer of matter, energy, and entropy across thin boundaries, by forcing relevant thermodynamic variables to adhere to a strict predetermined time dependence. As a prelude to this approach, detailed in earlier papers (Hoehn and Honig, 2011) (Honig and Hoehn, 2011), it was necessary to augment the conventional approach to the first and second laws through inclusion of irreversible processes. The aim of the present paper is to review this particular methodology. The theory, outlined below, certainly has antecedents cited in de Groot and Mazur (1962) and Gyarmati (1970), but those theoretical studies focused more on what is now termed extended thermodynamics, in which emphasis is placed on the identification of fluxes and thermodynamic forces in relation to steady state conditions, rather than on the time dependent phenomena studied in our earlier publications. Contributions in the literature directly relevant to the current investigation will be referred to at appropriate places. The author is not aware of any prior unified discussion of the material covered in the present review.

\section{Preliminaries}

The universe under study consists of a system that is characterized by temperature, pressure, and chemical potentials that differ from the corresponding intensive variables of a reservoir to which the system is anchored via a thin interlayer, as depicted in Fig. 1. For purposes of illustration the diagram depicts the temperature profile 
under consideration: all processes are assumed to occur sufficiently slowly that the system temperature remains uniform throughout; the temperature $T$ then changes smoothly but abruptly over the extension of the separation barrier until it assumes the uniform value $T_{0}$ of the system. This uniformity assumption restricts us to the consideration of quasistatic irreversible (QSI) phenomena, i.e., conditions not too far removed from reversibility, so as to avoid excesses such as turbulence, vortex formation, and the like. However, there is no restriction on the magnitude of the temperature difference. Similar considerations apply to other intensive variables, such as the pressure, or the various chemical potentials. The reservoir is assumed to be of such immense size and to remain so well mixed that its intensive variables remain unaltered in any of the processes considered below. Moreover, in conformity with standard practice, we assume that all events within the reservoir take place reversibly. The above uniformity restrictions on intensive variables may certainly be relaxed, but the resulting theory then becomes much more complex and obscures the fundamental principles developed below.

\section{Entropy Changes}

We begin with a study of entropy changes during an infinitesimal step of a particular process, which is first carried out reversibly. Designate the entropy change of the universe as $d S_{u}$, and let it be written as a sum of the entropy change $d S$ of the system and the entropy change $d_{a} S_{0}$ of the surroundings. Then, according to the Second Law no change is incurred in the entropy of the universe; thus

$$
d_{a} S_{u} \equiv d S+d_{a} S_{0} \quad=0 .
$$

Next, let the same step in the process within the system be carried out irreversibly. Since entropy is a function of state of the system, $d S$ is the same as before; however, the entropy change in the surroundings, designated by $d_{b} S_{0}$, is now different. The entropy change of the universe is then governed by the inequality

$$
d_{b} S_{u} \equiv d S+d_{b} S_{0}>0 .
$$

The use of inequalities is at best awkward and carries no information on the actual value of the variable of interest. Hence it is apposite to introduce an entropy deficit function $d \theta>0$ for the express purpose of converting Eq. (1b) into an equality, whereby it becomes possible to extend the standard methodology to irreversible phenomena:

$$
d_{b} S_{u} \equiv d S \quad+d_{b} S_{0} \quad-d \theta \quad=0 .
$$

At this stage the quantity $d \theta$ is simply a bookkeeping device. However, it becomes a meaningful quantity when we subtract (1a) from (1c) to obtain

$$
d_{b} S_{0}-d_{a} S_{0}=d \theta,
$$

which indicates that $d \theta$ represents the entropy transferred between the system and the surroundings when the infinitesimal step of interest in the system is carried out irreversibly as opposed to reversibly. Hence, a study of $d \theta$ becomes important in its own right.

We first restrict attention to heat exchanges taking place as a result of the temperature difference between the system and the reservoir. For ease of visualization we assume that $T_{0}>T$, so that in an infinitesimal step in the isolated unit of system + surroundings, any heat $(d Q)$ gained by the system operating at temperature $T$ is lost by the surroundings $\left(d Q_{0}\right)$ operating at temperature $T_{0}$, whence $d Q=-d Q_{0}$.

During a reversible process $(r)$, where the surroundings are at temperature $T_{0}$ infinitesimally above $T$, the corresponding entropy change of the system is given by $d S \equiv d_{r} S=d_{r} Q / T$, whence, by (1a), the entropy change in the surroundings is given by

$$
-d_{a} S_{0}=d S=d_{r} Q / T
$$

For the same step executed irreversibly $(i)$, with the temperature of the system at $T$ and that of the surroundings at $T_{0}$, the resulting heat exchange $-d_{b} Q_{0}=d_{i} Q$ produces an entropy change in the surroundings specified by

$$
-d_{b} S_{0}=-d_{b} Q_{0} / T_{0}=d_{i} Q / T_{0},
$$

where we invoked the reversibility of all processes in the reservoir. On inserting Eqs. (2a) and (2b) into Eq. (1d) we obtain the fundamental relation

$$
d_{i} Q / T_{0}=d_{r} Q / T-d \theta .
$$

or its equivalent

$$
d_{i} Q=\left(T_{0} / T\right) d_{r} Q-T_{0} d \theta=T_{0}(d S-d \theta)
$$




$$
\equiv d_{r} Q+\left(T_{0} / T-1\right) d_{r} Q-T_{0} d
$$

which now relates the heat transferred irreversibly in the infinitesimal step to that transferred reversibly. Eq. (3b) duplicates the expression derived by a different method (Tolman and Fine 1948). Eq. (3a) clearly shows that

$$
d_{i} Q<\left(T_{0} / T\right) d_{r} Q=T_{0} d S,
$$

and in the event that the reservoir temperature approaches that of the system we obtain a more restrictive inequality

$$
d_{i} Q<d_{r} Q=T d S,
$$

which is the famous Clausius inequality. It applies when a reversible heat transfer triggers irreversible processes totally with the system; Eq. (3d) is its generalization, arising from the double inequality $d_{i} Q<d_{r} Q<\left(T_{0} / T\right) d_{r} Q$.

In view of the requirement (3e) - which automatically allows for (3d) - the sum of the last two terms in Eq. (3c) must be negative:

$$
\left(T_{0} / T-1\right) d_{r} Q-T_{0} d \theta<0 .
$$

Careful examination shows that the above relations still apply when $T>T_{0}$, whereby heat is transferred from the system to the reservoir: in this event $d_{i} Q$ is more negative than $d_{r} Q$, which is more negative than $\left(T_{0} / T\right) d_{r} \mathrm{Q}$. Furthermore, the inequality (3f) remains unaltered: when $d_{r} Q<0$ the factor $\left(T_{0} / T-1\right)$ is also negative.

Eq. (3b) may be turned around to read

$$
d S=d_{i} Q / T_{0}+d \theta>d_{i} Q / T_{0},
$$

Thus, as is well known, in irreversible phenomena the total entropy change in the system is only partially compensated for by the transfer of heat across its boundaries, the remainder being attributed to the irreversibility effects totally within the system. Observe that it is the well-defined temperature of the reservoir that enters this relation. This should be contrasted with the usual expression $d S=d_{r} Q / T$, where the entropy change is tracked by the reversible heat transfer at the common temperature $T$. However, Eq. (3g) becomes useful only after methods are developed below that specify $d \theta$ in terms of experimentally measurable quantities, as shown below.

It is noteworthy that for processes that occur in isolation Eq. (3g) reduces to

$$
\delta S=d \theta>0,
$$

where the $\delta$ symbol serves as a reminder of the special conditions that prevail. As is well established, this equation shows that all processes proceeding without outside intervention (after release of a constraint) increase the entropy of the system. Such changes take place irreversibly since they are unaffected by outside manipulations. Successive increments produce a monotonic entropy increase until a state of maximum entropy is reached when the spontaneous process has run its course, at which point $\delta S=0$. This is the content of the famous dictum by Clausius that the entropy of the world (nowadays, we would say, of our universe) tends toward a maximum.

\section{Performance of Work}

At this point we introduce the First Law of Thermodynamics according to which the energy $E$ is a function of state. Then

$$
d E=d_{r} Q+d_{r} W=d_{i} Q+d_{i} W,
$$

where $W$ is the work performed during the process. On eliminating $d_{i} Q$ between (3c) and (4a) we may solve for

$$
d_{i} W=d_{r} W-\left(T_{0} / T-1\right) d_{r} Q \quad+T_{0} d \theta=d_{r} W-\left(T_{0}-T\right) d S+T_{0} d \theta .
$$

The last two terms in Eq. (4b) differ in sign from those cited in Eq. (3f). It follows that

$$
d_{i} W>d_{r} W
$$

When the process is carried out under conditions where $T$ and $T_{0}$ differ only infinitesimally,

$$
d_{i} W=d_{r} W+T_{0} d \theta,
$$

so that $T_{0} d \theta$ may then also be interpreted as the infinitesimal difference in work performance under reversible, as opposed to irreversible conditions, where additional phenomena take place as a result of the irreversible heat transfer. 
The above results are well known and accord with intuition. In any (infinitesimal) step the work performance is independent of the mode of operation. That is, in a given change $d E$ in energy of the system it does not matter whether this process is executed in terms of mechanical, electric, magnetic, or as any other type of work. Taking gravitational work as an example, it is intuitively clear that in irreversibly lifting a weight against the force of gravity over a given distance, more work is expended than in doing this reversibly. Hence, more generally in accord with Eq. (4d), for any given process we may set $d_{i} W>d_{r} W$ no matter what type of work is performed. This inequality holds even when both quantities are negative: the system performs less useful work in an irreversible than in a reversible step (i.e., $d_{i} W$ is less negative than $d_{r} W$ ). In view of (4c) or (3f), Eq. (4b) leads to an important inequality:

$$
d \theta>\left(1-T / T_{0}\right) d S
$$

Here the right hand side is always positive, as explained earlier. Thus, $d \theta$ has as its lower nontrivial bound the quantity on the right, which exceeds zero when the temperature of the system differs from that of the surroundings. Eq. (4e) provides useful information on $d \theta$ in terms of experimentally determinable variables $T$, $T_{0}$, and $d S$.

\section{Specification of the Deficit Function}

A very significant feature of the present analysis is manifested by solving Eq. (4b) for

$$
d \theta=\left(d_{i} W-d_{r} W\right) / T_{0}+\left(1-T / T_{0}\right) d S .
$$

This relation allows one to determine the contribution of irreversible phenomena to the infinitesimal entropy change of any process (subject to the constraints introduced earlier) in terms of quantities that may be measured or calculated. This requires monitoring the change in entropy of the system while incrementally carrying out a given process, taking due account of the change in temperature $T$; heat capacity measurements may be used for this purpose. Also, one needs to determine the total work required for executing the same change reversibly and irreversibly, equivalent to noting by how much a given weight changes height in the earth's gravitational field in the two processes.

We note that in the absence of work performance Eq. (5a) reduces to

$$
d \theta=\left(1 / T-1 / T_{0}\right) d_{r} Q,
$$

which was derived elsewhere by different means (Kestin, 1966). The first term in Eq. (5a) was obtained by a different approach (Bejan, 1997). Eq. (5a) further provides a thermodynamic background for the theoretical work of Jarzynski (1997) who related the average of repeated executions of irreversible microscopic processes to thermodynamic equilibrium processes. This work was confined to processes executed at constant temperature; the above relation shows how this analysis may be extended.

We next consider alternative methods for specifying $d \theta$.

\section{Functions of State}

So far we have discussed the properties of path-dependent quantities. We now turn to the development of functions of state under non-equilibrium conditions. This generalizes the conventional development of state functions.

\subsection{The Energy}

We begin with a consideration of the energy of a system. For this purpose we first apply the First Law of Thermodynamics to the surroundings, whose properties are all designated by the subscript zero. For an open system the energy differential under the assumed reversible operating conditions is specified by

$$
d E_{0}=T_{0} d S_{0}-P_{0} d V_{0}+\Sigma_{i} \mu_{0 i} d n_{0 i}
$$

where $P_{0}$ is the prevailing pressure, $V_{0}$ the volume, $\mu_{0 i}$ the chemical potential of species $i$, and $n_{0 i}$ is the corresponding mole number, all in the surroundings. Let the closed unit of system + surroundings be maintained at a constant volume. In that event, when the isolated unit undergoes irreversible processes, the system is subject to the constraints $d E=-d E_{0}, d S_{0} \equiv d_{b} S_{0}=-d S+d \theta$ (See Eq. (1c)), $d V_{0}=-d V$, and $d n_{0 i}=-d n_{i}$, so that the energy differential of the system is given as

$$
d E=T_{0} d S-P_{0} d V+\Sigma_{i} \mu_{0 i} d n_{i}-T_{0} d \theta .
$$

This relation, involving different arguments, was derived by Kestin (1966). The intensive variables appearing here are those of the surroundings and are therefore well defined, even when processes within the system are 
irreversible. The extensive quantities $d S, d V, d n_{i}$ are the control variables for the energy of the system; $d \theta$ is determined as shown in Eq. (5) or by the expressions developed below. Hence, $d E$ is well defined in the QSI procedure even under non-equilibrium operating conditions. If work other than mechanical is involved, appropriate terms must be added to Eq. (6b) as a product of intensive variables appropriate to the relevant work reservoir, and corresponding extensive variables for the system proper.

It is instructive to rewrite Eq. (6b) in the form

$$
d E=\left(T_{0}-T\right) d S-\left(P_{0}-P\right) d V+\Sigma_{i}\left(\mu_{0 i}-\mu_{i}\right) d n_{i}+T d S-P d V+\Sigma_{i} \mu_{i} d n_{i}-T_{0} d \theta,
$$

where the variables lacking the subscript zero refer to the properties of the system. Eq. (6c) is the generalized version of the conventional energy differential. Two special situations are of interest: Case (a). The infinitesimal step is executed reversibly, with $T=T_{0}, P=P_{0}, \mu_{0 i}=\mu_{i}$ and $d \theta=0$; then (6c) reduces to the standard form

$$
d E=T d S-P d V .+\Sigma_{i} \mu_{i} d n_{i} .
$$

Case (b). Here one removes the system slightly but irreversibly from its equilibrium state with $d S=d V=d n_{i}=0$ (all $i$ ) by external intervention, then removing the interference and allowing the system to respond under these constraints. This process is characterized via the relation $(6 \mathrm{~b})$ :

$$
\delta E=-T_{0} d \theta,
$$

where the $\delta$ symbol serves as a reminder of the special conditions under which this probing process is carried out. Thus, in the absence of normal driving processes any ongoing process occurs purely internally, while not being subject to experimental control. Thus, with $d \theta>0$ the energy of the system will spontaneously diminish in this step. On generalizing to finite processes, the energy of the system will continue to decrease until a state is reached where the spontaneous process ceases: then $\delta E=0$, and the energy of the system has reached a minimum consistent with the applied constraints. This is a statement that the energy of a system undergoing spontaneous processes will decrease until an equilibrium configuration has been reached.

Lastly since $E$ is a function of state we may subtract (6d) from (6c) to obtain

$$
T_{0} d \theta=\left(T_{0}-T\right) d S-\left(P_{0}-P\right) d V+\Sigma_{i}\left(\mu_{0 i}-\mu_{i}\right) d n_{i} .
$$

Since the quantities on the right are experimentally accessible the above equation permits the determination of $d \theta$ in terms of $S, V$, and $n_{i}$ as the control variables.

However, the above quantities are not generally the ones used in experiments; we next develop alternative expressions for $d \theta$ in terms of more readily accessible control parameters.

\subsection{The Helmholtz Energy}

In a similar manner we can construct the Helmholtz energy by introducing the definition $A=E-T S$, which sensibly involves the temperature $T$ of the system. This transforms the independent variable from $S$ to $T$. Using Eq. (6c) we then find that

$$
d A=\left(T_{0}-T\right) d S \quad-\left(P_{0}-P\right) d V+\Sigma_{i}\left(\mu_{0 i}-\mu_{i}\right) d n_{i}-S d T \quad-P d V+\Sigma_{i} \mu_{i} d n_{i}-T_{0} d \theta .
$$

However, since $A=A\left(T, V, n_{i}\right)$, the control variables for the Helmholtz energy are supposed to be temperature, volume, and composition, whereas Eq. (7a) involves a mix of variables. As a remedy we express the entropy in terms of the applicable control variables by setting $S=S\left(T, V, n_{i}\right)$. This yields the entropy in the appropriate differential form

$$
d S=(\partial S / \partial T)_{V, n_{i}} d T+(\partial S / \partial V)_{T, n_{i}} d V+\Sigma_{i}\left(\partial S / \partial n_{i}\right)_{T, V,} n_{i \neq j} d n_{i}
$$

Substitution in (7a) then produces the expression:

$$
\begin{gathered}
d A=\left(T_{0}-T\right)\left[(\partial S / \partial T)_{V, n_{i}} d T+(\partial S / \partial V)_{T, n_{i}} d V+\Sigma_{i}\left(\partial S / \partial n_{i}\right)_{T, V,} n_{i \neq j} d n_{i}\right]-\left(P_{0}-P\right) d V \\
+\Sigma_{i}\left(\mu_{0 i}-\mu_{i}\right) d n_{i}-S d T-P d V+\Sigma_{i} \mu_{i} d n_{i}-T_{0} d \theta .
\end{gathered}
$$

The partial differentials in the above relation may be rewritten in the following manner: $(\partial S / \partial T)_{V, n_{i}}=C_{V} / T$, and $(\partial S / \partial V)_{T, n_{i}}=(\partial P / \partial T)_{V, n_{i}}$, where $C_{V}$ is the heat capacity at constant volume and composition, and where the 
appropriate Maxwell relation has been introduced. Also, we use the mathematical identity $(\partial P / \partial T)_{V, n_{i}}=-(\partial V / \partial T)_{P, n_{i}} /(\partial V / \partial P)_{T, n_{i}}$ and then replace the numerator and denominator by $-\alpha V$ and by $\beta V$, where $\alpha$ and $\beta$ are the isobaric coefficient of expansion and the isothermal compressibility respectively. We also set $\left(\partial S / \partial n_{i}\right)_{T, V, n_{j \neq i}} \equiv \widehat{S}_{i}$ as the differential entropy at constant temperature, volume and composition of species $i$. In this notation,

$$
\begin{aligned}
d A \quad & \left(T_{0}-T\right)\left[\left(C_{V} / T\right) d T+(\alpha / \beta) d V+\Sigma_{i} \widehat{S}_{i} d n_{i}\right]-\left(P_{0}-P\right) d V \\
& +\Sigma_{i}\left(\mu_{0 i}-\mu_{i}\right) d n_{i}-S d T-P d V+\Sigma_{i} \mu_{i} d n_{i}-T_{0} d \theta
\end{aligned}
$$

The above equation specifies the differential of the Helmholtz function under non-equilibrium conditions in terms of $T, V$, and the $n_{i}$. The associated coefficients involve measurable quantities.

Under constraint (a) in which a reversible process is carried out with $T=T_{0}, P=P_{0}, \mu_{i}=\mu_{0 i}$, and $d \theta=0$, the Helmholtz function reduces to the standard form

$$
d A=-S d T-P d V+\Sigma_{i} \mu_{i} d n_{i} .
$$

Under constraint (b) we deform the system by external intervention under the conditions $d T=d V=d n_{i}=0$ to an adjacent state, and then release the system under these conditions. By analogy to the preceding discussion the resulting process will again be spontaneous and subject to the relation

$$
\delta A=-T_{0} d \theta,
$$

which shows that in a spontaneous process the Helmholtz energy diminishes and reaches a minimum at equilibrium, consistent with the indicated constraints.

Since $A$ is a function of state we may subtract (7e) from (7d) to find

$$
T_{0} d \theta=\left(T_{0}-T\right)\left[\left(C_{V} / T\right) d T+(\alpha / \beta) d V+\Sigma_{i} \widehat{S}_{i} d n_{i}\right]-\left(P_{0}-P\right) d V+\Sigma_{i}\left(\mu_{0 i}-\mu_{i}\right) d n_{i},
$$

which provides a second scheme for determining the entropy deficit function, here in terms of $T, V$, and $n_{i}$ as relevant experimental variables; all the coefficients involve measurable quantities.

\subsection{The Gibbs Energy}

Using by now familiar methodology, we define the Gibbs energy by the relation $G=E-T S+P V$. When converted to differential form, and on insertion of Eq. (7a), we obtain

$$
d G=\left(T_{0}-T\right) d S-\left(P_{0}-P\right) d V+\Sigma_{i}\left(\mu_{0 i}-\mu_{i}\right) d n_{i}-S d T+V d P+\Sigma_{i} \mu_{i} d n_{i}-T_{0} d \theta
$$

as the expression that holds under non-equilibrium conditions. However, the control variables in present circumstances should be $T, P$, and composition. Therefore, following the above recipe, we must express the entropy in the form $S=S\left(T, P, n_{i}\right)$ and the volume as $V=V\left(T, P, n_{i}\right)$, with corresponding differentials for $d S$ and $d V$. On inserting these in (8a) we obtain

$$
\begin{gathered}
d G=\left(T_{0}-T\right)\left[(\partial S / \partial T)_{P, n_{i}} d T+(\partial S / \partial P)_{T, n_{i}} d P+\Sigma_{i}\left(\partial S / \partial n_{i}\right)_{T, P,} n_{i \neq j} d n_{i}\right] \\
-\left(P_{0}-P\right)\left[(\partial V / \partial T)_{P, n_{i}} d T+(\partial V / \partial P)_{T, n_{i}} d P+\Sigma_{i}\left(\partial V / \partial n_{i}\right)_{T, P,} n_{i \neq j} d n_{i}\right]+\Sigma_{i}\left(\mu_{0 i}-\mu_{i}\right) d n_{i} \\
-S d T+V d P+\Sigma_{i} \mu_{i} d n_{i}-T_{0} d \theta .
\end{gathered}
$$

We next introduce the relations $(\partial S / \partial T)_{P, n_{i}}=C_{P} / T$ and the Maxwell relation $(\partial S / \partial P)_{T, n_{i}}=-(\partial V / \partial T)_{P,} n_{i}$. As before, the partial derivatives - $(\partial V / \partial P)_{T, n_{i}}$ and $(\partial V / \partial T)_{P, n_{i}}$ are specified by $\beta V$ and by $\alpha V$ respectively. $\left(\partial S / \partial n_{i}\right)_{T, P,} n_{i \neq j}$ and $\left(\partial V / \partial n_{i}\right)_{T, P,} n_{i \neq j}$ represent partial molal entropies $\bar{S}_{i}$ and volumes $\bar{V}_{i}$. We then rewrite Eq. (8b) in the less unwieldy form

$$
d G=\left(T_{0}-T\right)\left[\left(C_{P} / T\right) d T-\alpha V d P+\Sigma_{i} \bar{S}_{i} d n_{i}\right]-\left(P_{0}-P\right)[\alpha V d T
$$




$$
\left.-\beta V d P+\Sigma_{i} \bar{V}_{i} d n_{i}\right]+\Sigma_{i}\left(\mu_{0 i}-\mu_{i}\right) d n_{i}-S d T+V d P+\Sigma_{i} \mu_{i} d n_{i}-T_{0} d \theta .
$$

Eq. (8c) is a generalization of the conventional Gibbs function which holds under non-equilibrium conditions.

We once more consider two cases. Under alternative (a) we obtain the standard form

$$
d G=-S d T+V d P+\Sigma_{i} \mu_{i} d n_{i} .
$$

Under alternative (b) we contemplate a displacement of the system in the familiar manner from its equilibrium value to an adjacent state under the constraints $d T=d P=d n_{i}=0$, and then allow the system to undergo a spontaneous process back to the equilibrium state. This leads to the relation

$$
\delta G=-T_{0} d \theta,
$$

which shows that the Gibbs energy at equilibrium is a minimum under the indicated constraints.

Note further that when $(8 \mathrm{~d})$ is subtracted from $(8 \mathrm{c})$ we obtain

$$
\begin{gathered}
T_{0} d \theta=\left(T_{0}-T\right)\left[\left(C_{P} / T\right) d T-\alpha V d P+\Sigma_{i} \bar{S}_{i} d n_{i}\right]-\quad\left(P_{0}-P\right)[\alpha V d T \\
\left.-\beta V d P+\Sigma_{i} \bar{V}_{i} d n_{i}\right]+\Sigma_{i}\left(\mu_{0 i}-\mu_{i}\right) d n_{i}
\end{gathered}
$$

which shows how one may determine the deficit function when $T, P$, and composition are the independent variables.

\subsection{The Enthalpy}

Lastly, we turn to the enthalpy $H=E+P V$. By the customary technique we develop the differential form $d H=$ $d E+P d V+V d P$ and then insert Eq. (6c). We obtain

$$
d H=\left(T_{0}-T\right) d S-\left(P_{0}-P\right) d V+\Sigma_{i}\left(\mu_{0 i}-\mu_{i}\right) d n_{i}+T d S+V d P+\Sigma \mu_{i} d n_{i}-T_{0} d \theta .
$$

Since $H=H\left(S, P, n_{i}\right)$, it is $S, P$, and composition that are regarded as control variables. We therefore consider the volume first in the form $V=V\left(P, T, n_{i}\right)$. We next introduce the entropy as a function of the same variables: $S$ $=S\left(P, T \cdot n_{i}\right)$, which function we invert to read $T=T\left(S, P, n_{i}\right)$. Lastly, we insert this expression into the equation of state: thus,

$$
V=V\left(P, T\left(S, P, n_{i}\right), n_{i}\right) \equiv V\left(S, P, n_{i}\right) .
$$

On taking the differential of this latter relation we find

$$
d V=(\partial V / \partial S)_{P, n_{i}} d S+(\partial V / \partial P)_{S, n_{i}} d P+\left(\partial V / \partial n_{i}\right)_{S, P, n_{i \neq j}} d n_{i}
$$

Then, substituting (9b) into (9a) we obtain

$$
\begin{aligned}
d H=\left(T_{0}-T\right) d S- & \left(P_{0}-P\right)\left[(\partial V / \partial S)_{P, n_{i}} d S+\quad(\partial V / \partial P)_{S, n_{i}} d P+\quad\left(\partial V / \partial n_{i}\right)_{S, P, n_{i \neq j}} d n_{i}\right] \\
+ & \Sigma_{i}\left(\mu_{0 i}-\mu_{i}\right) d n_{i}+T d S+V d P+\Sigma_{i} \mu_{i} d n_{i}-T_{0} d \theta .
\end{aligned}
$$

This specifies the enthalpy change in an infinitesimal step under nonequilibrium conditions. Under constraint (a) Eq. (9b) reduces to the standard form

$$
d H=T d S+V d P+\Sigma_{i} \mu_{i} d n_{i},
$$

while under constraint (b), with $d P=d S=d n_{i}=0$ we obtain the result

$$
\delta H=-T_{0} d \theta,
$$

where again we noted that the enthalpy change in such a spontaneous process is a minimum under equilibrium conditions, subject to the relevant constraints.

When $(9 \mathrm{~d})$ is subtracted from $(9 \mathrm{c})$ we find that 


$$
\begin{gathered}
T_{0} d \theta=\left(T_{0}-T\right) d S-\left(P_{0}-P\right)\left[(\partial V / \partial S)_{P, n_{i}} d S+(\partial V / \partial P)_{S, n_{i}} d P+\Sigma_{i}\left(\partial V / \partial n_{i}\right)_{S, P,} n_{i \neq j} d n_{i}\right] \\
+\Sigma_{i}\left(\mu_{0 i}-\mu_{i}\right) d n_{i},
\end{gathered}
$$

which yields yet another formulation for the deficit function that holds when $S, P$, and $n_{i}$ are the variables of interest.

The minima achieved by the above functions of state should be contrasted with the maximum that characterizes the entropy of an isolated system undergoing spontaneous processes.

\section{Concluding Remarks}

Complementary to the standard methodology of irreversible thermodynamics the above approach focuses on extending the conventional functions of state so as to include contributions arising from irreversible processes. In particular, their contribution to the entropy of a system has been specified in terms of applicable control variables. This serves as a basis for dealing with exchange of matter, energy, and entropy across a narrow interface between a system and its surroundings under non-equilibrium conditions. As shown elsewhere (Hoehn and Honig, 2011; Honig and Hoehn, 2011), the actual determination of the entropy is carried out by specifying the time dependence of all applicable control variables and then integrating the relevant relation involving $d \theta$, namely (6f), (7g), (8f), or (9f). The principal restriction, in common with standard procedures, is that the changes must occur sufficiently slowly that all intensive quantities in the system change uniformly, but there is no limit set on the difference between these quantities and those of the reservoir to which the system is anchored. Explicit relations have been provided, loc. cit., which allow one to determine the contribution of irreversible processes to the entropy change of a system under prescribed conditions, based on the use of Eqs. (8f) and (7g).

It is hoped that the present treatment provides further insights on how to deal with irreversible phenomena within the framework of equilibrium thermodynamics.

\section{Acknowledgments}

The author wishes to acknowledge with gratitude the many fruitful conversations with his collaborator, Professor Dor Ben Amotz, on the topics dealt with in this article.

\section{References}

Bejan A. (1997). Advanced Engineering Thermodynamics, 2nd Ed. Wiley, New York, p 135.

Ben Amotz D. \& Honig J.M. (2006). The Rectified Second Law of Thermodynamics. J. Phys. Chem. B, 110, pp 19966-19972. http://dx.doi.org/10.1021/jp0621631

Ben Amotz D. \& Honig J.M. (2006). Average Entropy Dissipation in Irreversible Mesoscopic Processes. Phys. Rev. Lett., 96, pp 020602/1-4. http://dx.doi.org/10.1103/PhysRevLett.96.020602

de Groot S.R. \& Mazur P. (1962). Non-Equilibrium Thermodynamics. North Holland, Amsterdam.

Gyarmati I. (1970). Non-equilibrium Thermodynamics, Field Theory and Variational Principles. Springer Verlag, New York.

Haase R. (1990). Thermodynamics of Irreversible Processes. Dover, New York.

Hoehn R. and Honig J.M. (2011). Analysis of Irreversible Processes across Narrow Junctions. Acta Phys. Polon. $A, 119$, pp 323-327.

Honig J.M. (2008). Thermodynamics, Principles Characterizing Chemical and Physical Processes, 3rd Ed., Academic Press, Amsterdam, Chap. 6.

Honig J.M. \& Ben Amotz D. (2005). The Analysis of Spontaneous Processes Using Equilibrium Thermodynamics. J. Chem. Ed., 83, pp 132-137. http://dx.doi.org/10.1021/ed083p132

Honig J.M. \& Ben Amotz D. (2008). Rectification of Thermodynamic Inequalities as a Means of Characterizing Irreversible Phenomena. Chem. Educator, 13, pp 220-226. http://dx.doi.org/10.1333/s00897082138a

Honig J.M. \& Hoehn R. (2011). Entropy of Irreversible Processes across a Boundary. Open J. Chem. Eng., 5, pp 1-6. http://dx.doi.org/10.2174/1874123101105010001

Jarzynski C. (1997). Non-Equilibrium Equality for Free Energy Differences. Phys. Rev. Lett., 78 pp 2690-2693. http://dx.doi.org/10.1103/PhysRevLett.78.2690

Jou D. (1996). Extended Irreversible Thermodynamics. Springer Verlag, New York. 
Kestin, J. (1966). A Course in Thermodynamics. Blaisdell, Waltham, MA, Chap. 13.

Kondepudi D \& Prigogine I. (1998). Modern Thermodynamics, from Heat Engines to Dissipative Structures, Wiley, Chichester. Chaps 15-17.

Meixner J. (1954). Thermodynamik der Irreversiblen Prozesse. Meizner, Aachen.

Mueller I. \& Ruggeri T. (1993). Extended Thermodynamics, Springer Verlag, New York.

Prigogine I. (1967). Introduction to Thermodynamics of Irreversible Processes. Wiley, New York.

Tolman R.C. \& Fine, P.C. (1948). On the Irreversible Production of Entropy. Rev. Mod. Phys., 20, pp 51-77 http://dx.doi.org/10.1103/RevModPhys.20.51

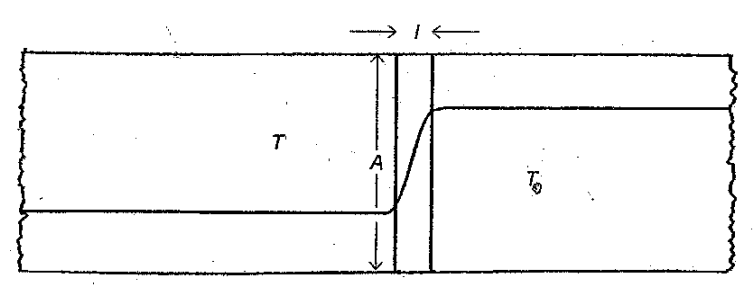

Figure 1. Temperature Profile for a System at temperature $T$ (left) Attached to a Reservoir at Temperature $T_{0}$ (right); the Temperature Difference is confined to a Narrow Region of Area $A$ and Length $l$. 